\title{
Digital Design as a Key Approach to Shortening MEMS Development Cycle
}

\author{
Mihail M. Andronic ${ }^{1 *}$, Ilya A. Rodionov ${ }^{1,2}$, and Yurii B. Tsvetkov ${ }^{1}$ \\ ${ }^{1}$ Bauman Moscow State Technical University, 2nd Baumanskaya str., 5/1, 105005, Moscow, Russia \\ ${ }^{2}$ Dukhov Automatics Research Institute (VNIIA), Sushchevskaya str., 22, 127055, Russia, Moscow, \\ Russia
}

\begin{abstract}
A comparative analysis of new product development principles in microelectronics, mechanical engineering, and MEMS production is carried out. A MEMS integrated digital modeling approach is proposed based on the formation of a knowledge base, including a description of basic structural elements and basic tested process sequences for their manufacture.
\end{abstract}

\section{Introduction}

The concept of the fourth industrial revolution (Industry 4.0) provides end-to-end digitization of all physical processes and their integration into the digital ecosystem. It corresponds to new digital technologies, the typical examples of which can be considered the Internet of things (IoT) and its industrial variety - the Industrial Internet of things (IIoT). IoT (Fig. 1) refers to a wide variety of devices, sensors, actuators, which are networked through communication channels.

\section{Industry 4.0}

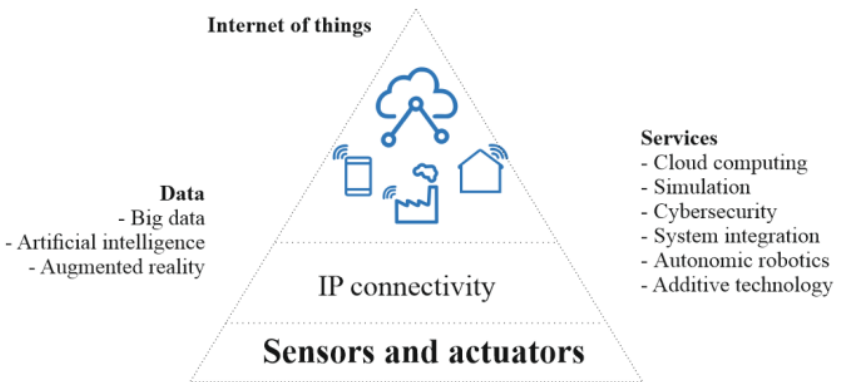

Fig. 1. Industrial transformation pyramid.

Designed to convert a signal from one physical form of energy to another, sensors and actuators play one of the main roles in such systems [1]. Sensors convert pressure, humidity, gas concentration, sound, acceleration, radiation, etc., into an electrical signal.

\footnotetext{
* Corresponding author: $\underline{m}$ andronic @ bmstu.ru
} 
Actuators respond to a change in the input signal by changing the useful optical, electrical signal, and are necessary for the organization of control systems, including temperature control, damping systems, and others.

Microelectromechanical systems (MEMS) are sensors and actuators which are made primarily on silicon wafers using group processing technologies adopted from the microelectronics technology (Fig. 2). Based on a unique combination of silicon semiconductor electrical and mechanical properties, MEMS technologies create a complex platform for a wide range of sensor and actuator applications. The rapid development of MEMS technologies is largely spread due to their key advantages: small size, functionality, low power consumption, ease of integration, and scalability to mass production. According to [2], RF switches (Fig. 3), oscillators, and microfluidic devices expect the highest growth rate. Other devices show positive growth rates, which indicates an expanding demand for these products.

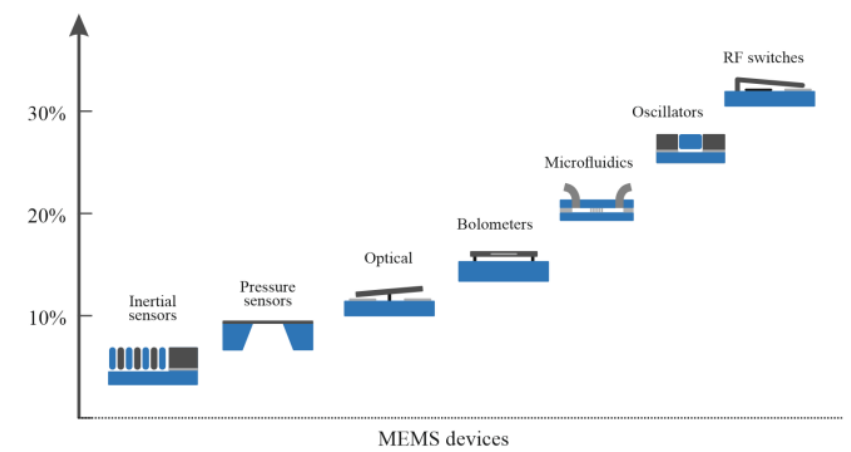

Fig. 2. The average annual growth rate of the main types of MEMS.

MEMS products have been successfully implemented in various areas of life and today they are replacing the macro-level sensors. The world is becoming more and more equipped with sensors and actuators, which monitor in real-time a large number of various processes and find applications in a wide variety of fields: from new high-end industries to the creation of a smart surrounding world. Depending on the consumer requirements (Fig. 3), the development and fabrication time and costs differ widely. Fig. 3. MEMS device applications
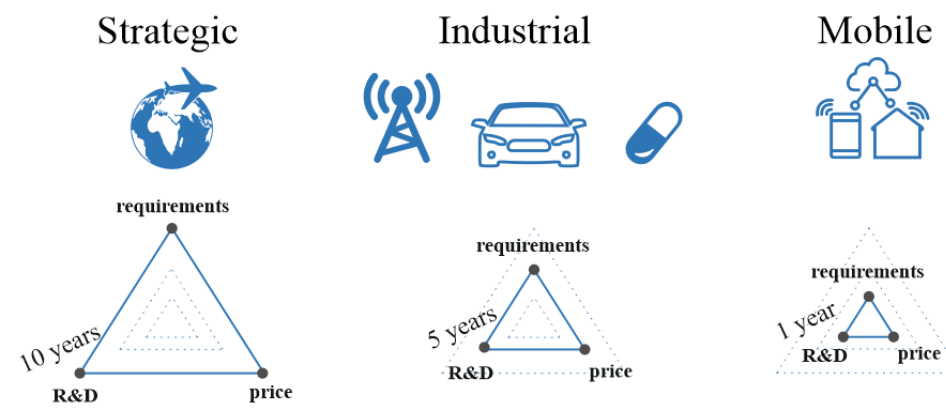

Fig. 3. MEMS device applications.

Already today, according to Yole Development [3], the contribution of the cost of sensors is about $5 \%$ of the final cost of the product, and by 2030 part of is expected to grow up to $30 \%$ of the cost of products. This is connected with the increasing needs of the Internet of things and the new paradigm of industrialization. 
The mass production of MEMS is based on the similarity of its technology with integrated circuit technology. The fabrication of MEMS in mass production is possible due to the process and equipment similarities with integrated circuits technologies. One of the most important features of MEMS is that their critical dimensions are greater than $1 \mu \mathrm{m}$ (Fig. 4), which makes it possible to use the relatively cheap contact lithography technology as opposed to the projection photolithography used in microelectronics technology.

\section{Microelectronics MEMS Mechanical engineering}

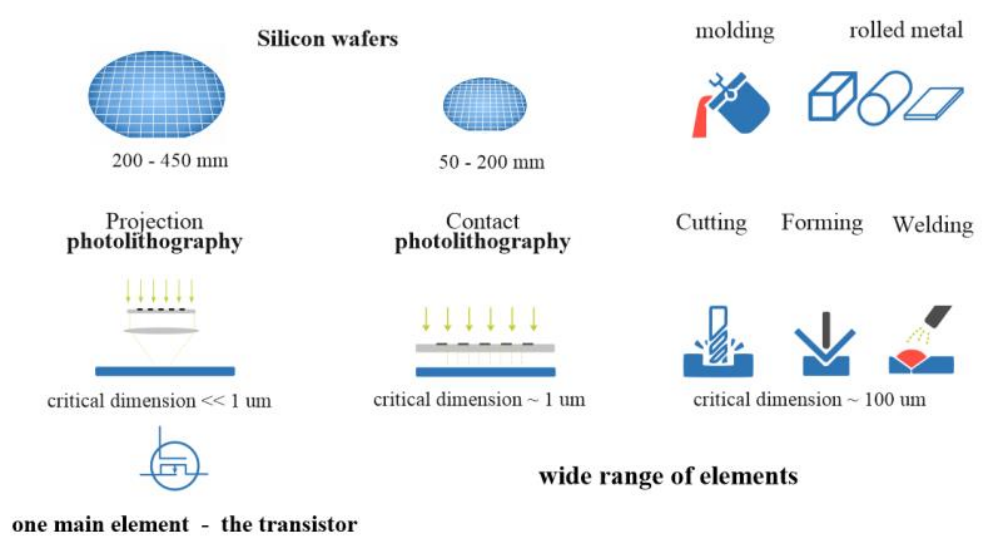

Fig. 4. MEMS at the intersection of two fields of science.

It is also interesting to reveal a deeper analysis of technologies and approaches for a new MEMS product development in comparison with microelectronics and mechanical engineering (instrument making) technologies.

\section{Development of new microelectronics products}

In the 1970s, integrated circuits (IC) manufacturing was more an "art", where each designer had to work closely with the people operating the fabrication line in order to define appropriate patterns that together with the envisioned processing sequence would then produce the desired circuit behavior. But already in 1980 [4] with the work "Introduction to VLSI systems", a new paradigm shift in this area took place. It consisted of the systematization, simplification, and standardization of the circuit elements, which allowed us to separate the technology and manufacturing process by a set of rules. At the same time, the geometry of the circuits (sizes, shape of elements and positioning restrictions), as well as the structural and functional behavior of the circuit were subjected to parallel analysis. This approach is illustrated by the Gajski-Kuhn Y-chart (Fig. 5) [5]. 


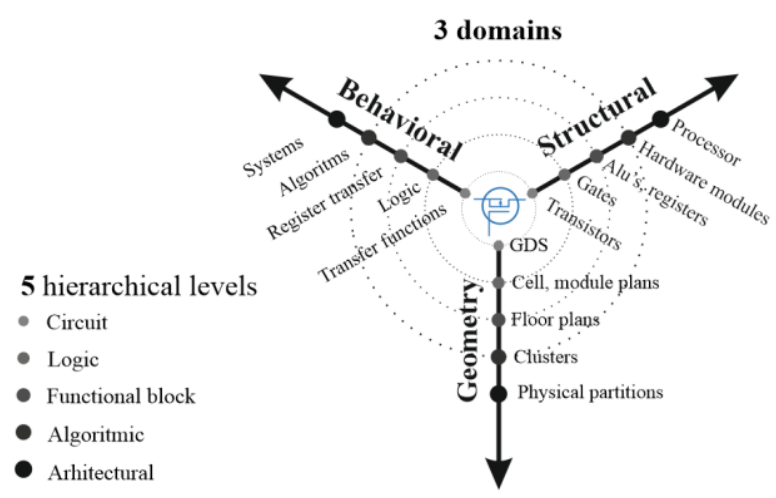

Fig. 5. Gajski-Kuhn Y-chart.

From a technology point of view, photolithography is a key process. For a relatively short period of 50 years, the minimum sizes of IC elements patterned by this method were decreased by 10,000 times - from $50 \mu \mathrm{m}$ to $5 \mathrm{~nm}$, which corresponds to Moore's law: every 2 years the number of transistors on a crystal doubles.

The technological process in microelectronics determines the possibility of manufacturing a particular chip, while the transition from one technological norm to another is associated with a qualitative leap in production, providing radical changes in the type of equipment and materials.

To ensure the need for design automation of microelectronic devices, a special software class was developed: Synopsys (1986, USA), Cadence (1988, USA), Mentor Graphics (1981, USA), Zuken Inc. (1976, Japan).

Thus, the characteristic features of the production of integrated circuits consist of one basic, standard (primitive) element, the technological norm as a determining factor in the possibility of manufacturing, as well as the formed multi-level hierarchical system with three areas of analysis. This allowed separating the work of designers and technologists due to the development of the design rules.

Figures and tables, as originals of good quality and well contrasted, are to be in their final form, ready for reproduction, pasted in the appropriate place in the text. Try to ensure that the size of the text in your figures is approximately the same size as the main text (10 point). Try to ensure that lines are no thinner than 0.25 point.

\section{Development of new products in mechanical and instrument engineering}

Mechanical engineering is a complex of industries producing tools for the national economy, vehicles, consumer goods, etc. This complex includes instrument making, manufacturing measuring instruments, information processing tools, control devices, automatic and automated control systems.

The complex of the main types of processing in mechanical and instrument engineering includes cutting, casting, pressing, stamping, welding, additive technologies, and manufacturing technologies for composite materials [6]. When developing a new product, the developer has complete freedom of choice among these types of processes. Product manufacturability mainly determines the cost of the product because is a complex parameter and is related simultaneously to the design of the product and the availability of equipment and processes. 
The production is regulated by GOST, ISO standards, which, together with interchangeability, makes it easier to design, manufacture, assemble and operate products.

Digital modeling is a key tool to reduce development time and is widely used to increase product development efficiency [7]. At the same time, the main objective of creating a new product is to satisfy the customer needs, reflecting as well the aim of the "Quality Factor Deployment" approach (hereinafter QFD) [8].

In accordance with this approach, the quality requirements are converted at the first stage (Fig. 6) into quantitative engineering metrics by arranging weight coefficients, which at the second stage of development are converted into requirements for product's part characteristics. The product design set restrictions on the possibility of using technological processes (third stage). At the fourth stage, the process sequence is optimized.

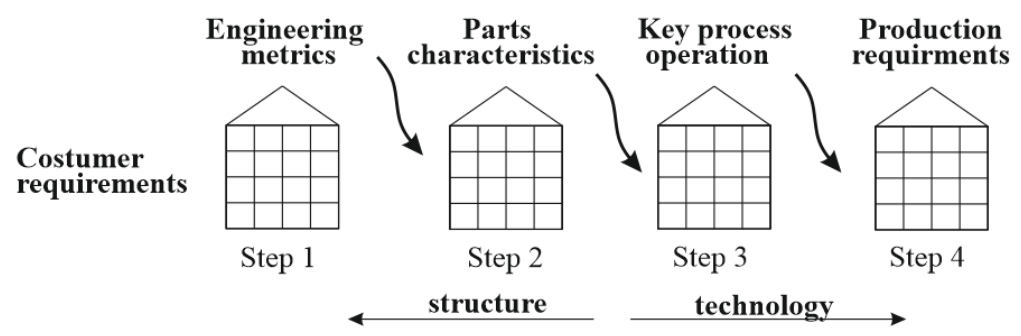

Fig. 6. Stages of the "Quality Factor Deployment" tool.

The complexity of this approach is compensated by its focus on achieving customer requirements. Also, QFD implementation avoids errors in the product design and manufacture at early stages.

Figure 7 shows schematically the first stage of quality factor deployment, expressing the relationship between the customer requirements and the engineering metrics through weighting coefficients. The accuracy of the weight coefficients that are filled in the matrix is determined by the synergy between managers' and engineers' experience and estimations.

The resulting weight coefficients between technical parameters and customer requirements go to the next stage to form the next house of quality (Fig. 7).

Apart from that, in this approach, the mutual influence of technical parameters is taken into account (Fig. 7, correlation matrix), since the required parameters can contradict each other in order to achieve them simultaneously. The detection and solution of such contradictions in the early stages of product development increase the efficiency of the process.

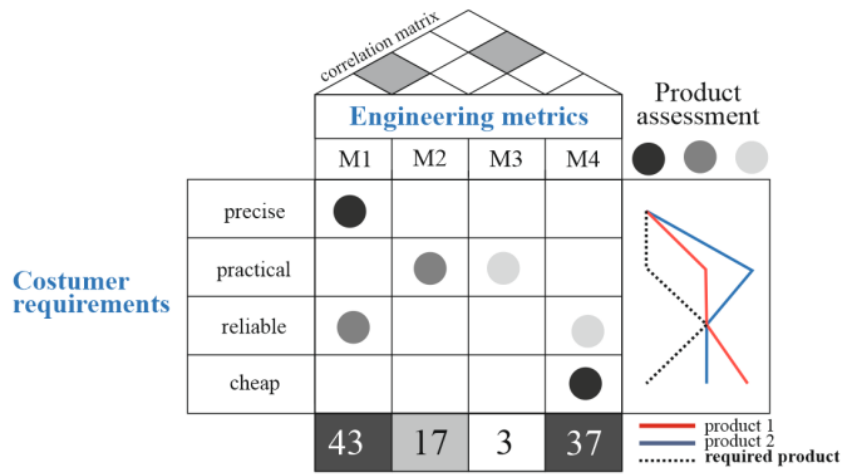

Fig. 7. The first stage of structuring the "Quality Factor Deployment" tool. 
The right part of figure 7 shows the correlation between the parameters of the new product (dashed line) and existing products (solid lines), which allows us to evaluate the uniqueness of the new product. A comprehensive overview of QFD is presented in [9], and more than 50 applications of this tool are structured in [10].

To solve the problem of choosing the manufacturing technology for a new product specialized software was created aimed at gathering information about materials and manufacturing processes that are presented in the form of structured databases [11]. This extends the developer available choices, allowing him or her to consider the influence of changing the design requirements on the available processes for manufacturing.

This consulting software class includes programs as Computer Aided Material and Process Selector (CAMPS, 1991), Manufacturing Analysis Service (1998), Cambridge Engineering Selector (CES, 1998). In [12] a comparative analysis of these programs was carried out according to the criteria of input data, available databases and operation algorithms.

Thus, a large selection of available technologies and an abundance of standard elements, are characteristic for the production of engineering and instrumentation products, while the choice of technology is determined by the design of the product.

\section{MEMS (microelectromechanical systems)}

The MEMS designing issue in comparison with integrated circuits design is that MEMS consists of many basic elements [13] that correspond to different physical domains: mechanical, electrical, optical, etc.

MEMS can be divided into 3 main categories by the degree of mobility of elements (Fig. 8): MEMS without mechanical displacements, MEMS with bending mechanical elements and mechanical systems with contacting elements: for the realization of electrical contact, or latching mechanisms, etc.

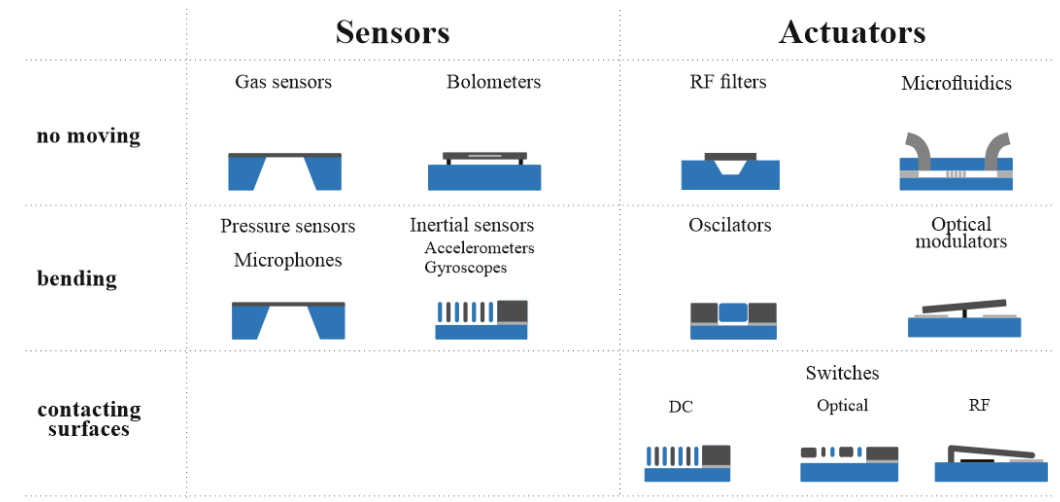

Fig. 8. Classification of main MEMS products.

This approach allows us to combine the set of standard elements in groups, which facilitates the description and modeling of such systems. It should be noted that the quality factor deployment approach was already tested in MEMS product development [14], and the idea of using digital modeling was described in [15].

There are a number of commercial MEMS design and simulation programs: IntelliSuite (1991), Tanner MEMS Design Flow (2004, Tanner Research), CoventorWare (1996, Lam Research) [16]. IntelliSuite begins the modeling process from the necessary parameters of the mechanism and provides tools for optimizing a variety of structures and tools for 
controlling the process sequence. Tanner MEMS Design Flow offers a topological editor optimized for MEMS with the ability to verify design and simulation rules. CoventorWare is designed to simulate inertial sensors, microphones, resonators, and RF switches.

MEMS modeling software is widely used in leading universities: MEMCAD (M.I.T., USA), CAEMEMS (Univ.of Michigan), CAPSIM (Catholic Univ. of Leuven, Belgium), SENSOR (Fraunhofer Institute, Germany), SESES (ETH, Zurich) [17]. As a rule, these universities have their own technological capabilities for manufacturing MEMS, which allows developers to quickly make prototypes of their devices.

In MEMS technology, one can distinguish groups of basic operations that provide layerby-layer formation of the necessary structure. These include surface substrate preparation, deposition of layers of materials (sputtering, PVD, CVD), photolithography, local etching, and several others.

When creating a process sequence, it is necessary to take into account various conflicting parameters, which is usually achieved due to engineering creativity. Silicon Deep Reactive Ion Etching (DRIE), chemical deposition of nitrides and oxides, alloying, anode bonding, etc. are considered standard operations in MEMS technologies.

The analysis of well-known MEMS processes sequences (Fig. 9), revealed that these process sequences consist of a limited common set of operations. In addition, each process typically includes operations with increased requirements. For example, the packaging operation in the SiTime process using the deposition of polycrystalline silicon, the fabrication of high-aspect structures from silicon in the HARPSS process, or the formation of a large membrane in the Microphone process.

\begin{tabular}{|c|c|c|c|c|c|c|c|c|c|c|c|c|c|c|c|c|c|c|c|}
\hline \multirow[b]{3}{*}{ Developer } & \multirow[b]{3}{*}{ Process sequence } & \multirow{2}{*}{\multicolumn{2}{|c|}{\begin{tabular}{|c|} 
Etching \\
$\mathrm{Si}$ \\
\end{tabular}}} & \multicolumn{3}{|c|}{ CVD } & \multirow[b]{3}{*}{ 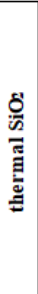 } & \multirow{2}{*}{\multicolumn{3}{|c|}{ Metals }} & \multirow[b]{3}{*}{ 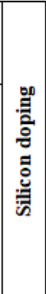 } & \multirow[b]{3}{*}{$\begin{array}{l}n \\
\mathbf{N} \\
\dot{z} \\
\bar{u}\end{array}$} & \multirow[b]{3}{*}{ 产 } & \multirow{2}{*}{\multicolumn{3}{|c|}{ Bonding }} & \multirow{2}{*}{\multicolumn{3}{|c|}{$\begin{array}{c}\text { Special } \\
\text { processes }\end{array}$}} \\
\hline & & & & \multirow{2}{*}{ 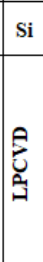 } & \multicolumn{2}{|c|}{$\begin{array}{l}\mathrm{SiO}_{2}, \\
\mathrm{Si}_{4} \mathrm{~N}_{4}\end{array}$} & & & & & & & & & & & & & \\
\hline & & 桃 & 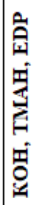 & & O & 畠 & & 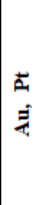 & 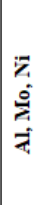 & 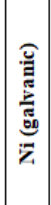 & & & & 苛 & :气 & 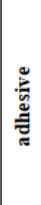 & 产 & 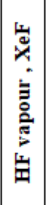 & 胞 \\
\hline Avago & FBAR & & & & & & & & & & & & & & & & & & \\
\hline Knowles & Microphone & & & & & & & & & & & & & & & & & & \\
\hline SiTime & Resonator & & & & & & & & & & & & & & & & & & \\
\hline Draper & Gyroscope & & & & & & & & & & & & & & & & & & \\
\hline STMicroelectronics & Thelma & & & & & & & & & & & & & & & & & & \\
\hline Novasensor & Pressure Sensor & & & & & & & & & & & & & & & & & & \\
\hline Ford Microelectronics & Bulk Accelerometer & & & & & & & & & & & & & & & & & & \\
\hline Infotonics & Hybrid MEMS & & & & & & & & & & & & & & & & & & \\
\hline MEMtronics & RF MEMS Switch & & & & & & & & & & & & & & & & & & \\
\hline Texas Instruments & DLP & & & & & & & & & & & & & & & & & & \\
\hline Xerox & Thermal Inkjet & & & & & & & & & & & & & & & & & & \\
\hline Honeywell & Microbolometer & & & & & & & & & & & & & & & & & & \\
\hline MEMSCap & PolyMUMPS & & & & & & & & & & & & & & & & & & \\
\hline MEMSCap & SOIMUMPS & & & & & & & & & & & & & & & & & & \\
\hline MEMSCap & MetalMUMPS & & & & & & & & & & & & & & & & & & \\
\hline Sandia & SUMMiT V & & & & & & & & & & & & & & & & & & \\
\hline Cornell University & SCREAM & & & & & & & & & & & & & & & & & & \\
\hline University of Michigan & HARPSS & & & & & & & & & & & & & & & & & & \\
\hline University of Michigan & Silicon-on-Glass & & & & & & & & & & & & & & & & & & \\
\hline University of Michigan & Plastic MEMS & & & & & & & & & & & & & & & & & & \\
\hline CAMD & LIGA & & & & & & & & & & & & & & & & & & \\
\hline
\end{tabular}

Fig. 9. Caption of the Figure 1. Below the figure.

The variety of materials and operations used in the development of the MEMS product process sequence (Fig. 9) face the problem of process integration and compatibility in a 
single process sequence, which mainly consists of taking into account the thermal budget, maximum allowable structures stress, selectivity of materials during etching, etc.

Despite the similarity between the operations and the used materials, the final structure of MEMS products differ significantly (Fig. 10), which is associated with a large range of MEMS functional elements. This indicates that the sequences of these technological processes are fundamentally different, while there is no universal manufacturing process for MEMS fabrication.

In general, companies prefer dedicated processes, as a rule, based on patented technologies and unique operations [18], which provide a competitive technological advantage. On the contrary, universities are characterized by the priority of universal processes that allow them to expand the range of manufactured products (Fig. 10).

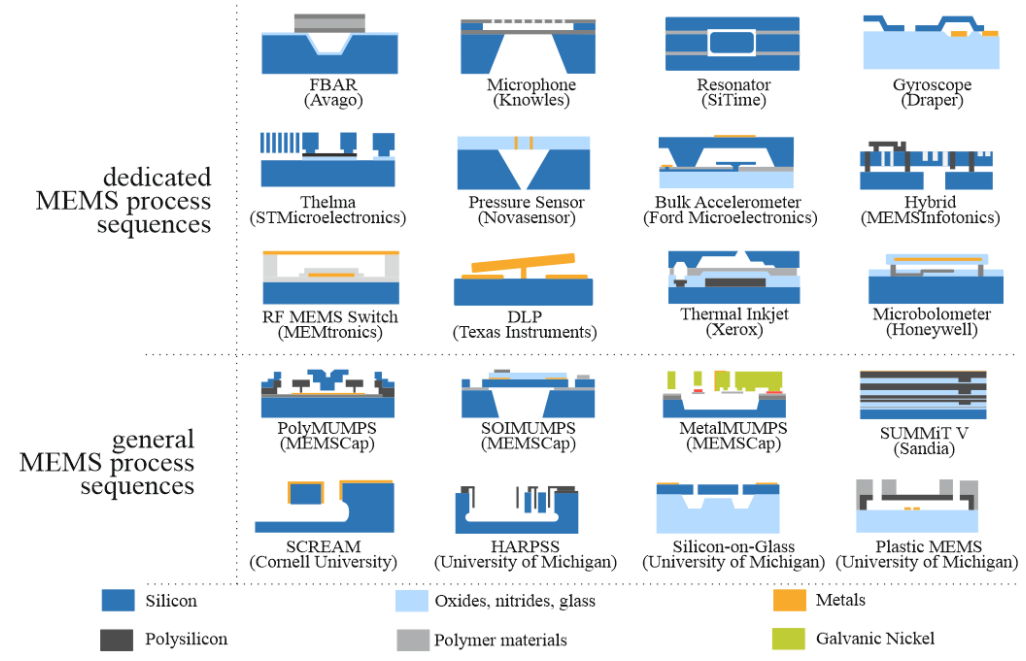

Fig. 10. An example of existing MEMS processes.

A clear comparison of mechanical engineering, microelectronics and MEMS as objects of design and manufacture shows a special place of MEMS in theses series (Fig.11). Many features were noted already a quarter-century ago [13] when the problem of replacing prototyping with the design rules was firstly highlighted.

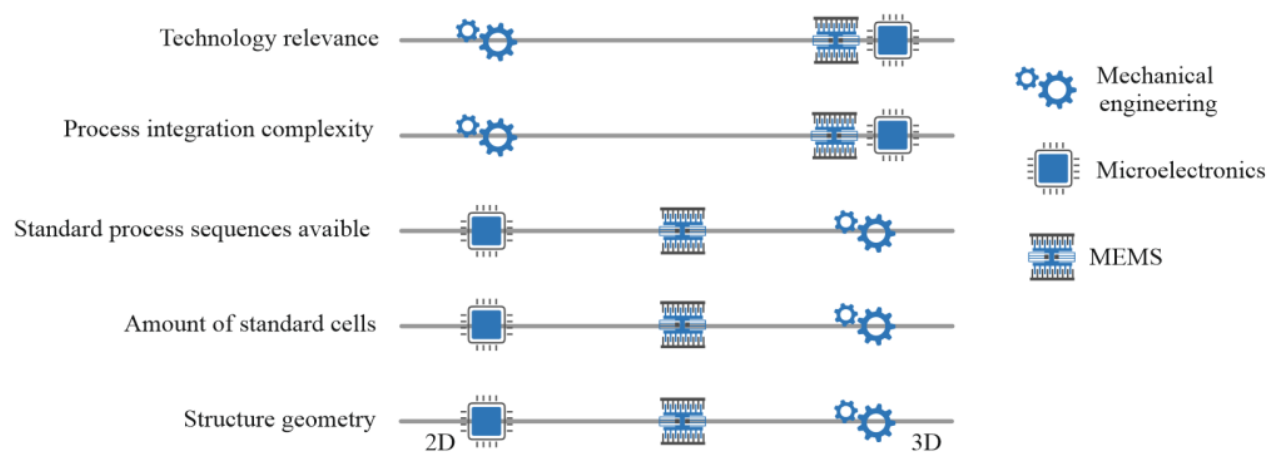

Fig. 11. Comparison of microelectronics, mechanical engineering and MEMS approaches. 
The obvious difference between MEMS technologies and integrated circuit technologies is the lack of multipliable basic elements in MEMS, and the realization of various physical principles in MEMS, which simultaneously require both mechanical and electrical properties. This explains the lack of a systematic approach at the stages of development and design of the MEMS technological process, the use of trial and error method, which often leads to unjustified loss of time and material resources. A relevant example is work [18] aimed at the creation of gyroscopes using different MEMS processes: PolyMUMPS, metalMUMPS, "Dissolved wafer", SOIMumps and Silicon on Glass, carried out for 5 years in order to achieve the required technical parameters.

The solution to this problem can be considered the creation of process knowledge bases along with operations and MEMS technologies databases. In this case, it is necessary to indicate the importance of identifying basic processes within which it is possible to create basic elements of a given MEMS class. In turn, we need to determine the necessary and sufficient set of basic elements that determine the possibility of designing the promising products.

The proposed approach (Fig. 12) provides a reduction in the MEMS product development cycle through the use of digital modeling and matching the developed product with the capabilities of available technologies and design solutions. In the classical approach (Fig. 12, a), in order to develop a process sequence, it is necessary to carry out several iterations of structural changes, key operations, and the manufacturing route of the sensor or actuator with the necessary parameters.

To reduce the development time, it is proposed to combine technology and design through the creation of a knowledge base of standard elements and sequences (Fig. 12, b). This approach allows us to increase the range of manufactured products, first of all, by using the already accumulated world scientific experience, and by accumulating the information about successfully manufactured devices, that as a result will reduce the duration and costs of development through the use of standard successfully tested processes.

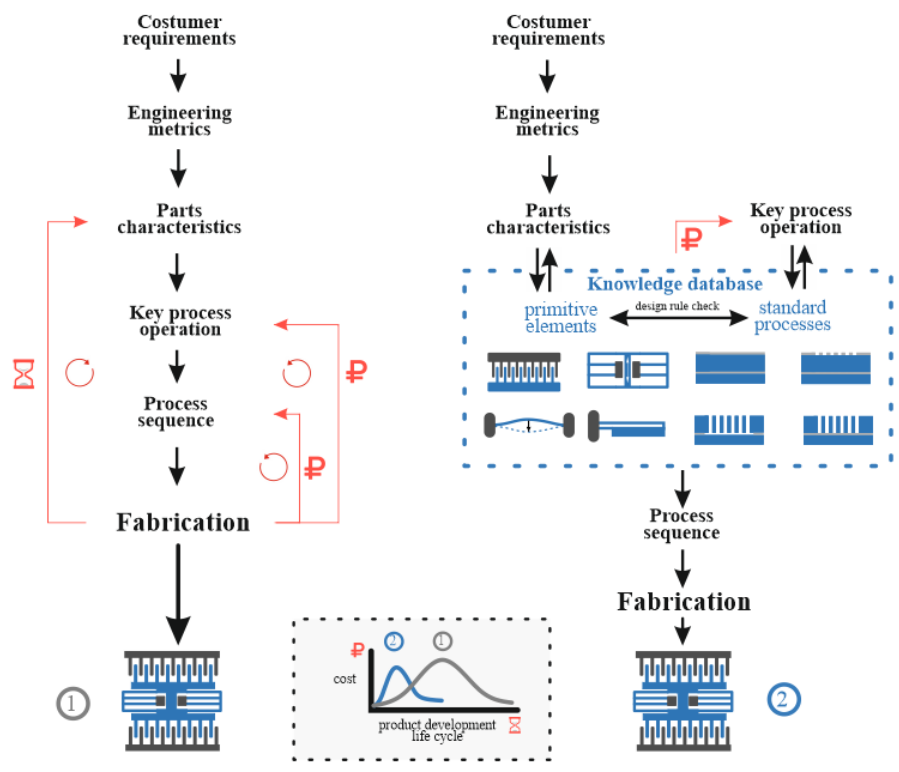

a) classic MEMS development approach

b) proposed MEMS development approach

Fig. 12. Approaches to the development of MEMS devices. 
The knowledge base (Fig. 13) expands the concept of the database because it combines information about the possibility of manufacturing along with the information about the structures of the developed product.

A standard element in terms of the knowledge base is a structural element that has an independent function determined by geometry, with reference to the properties of materials, as well as a mathematical model of its behavior and output parameters. A standard sequence is a process sequence consisting of trusted processes (proved to be compatible) for which a possible range of geometric dimensions of elements is determined and design rules are described.

Within the one standard process sequence, a variety of possible basic elements are feasible and vice-versa.

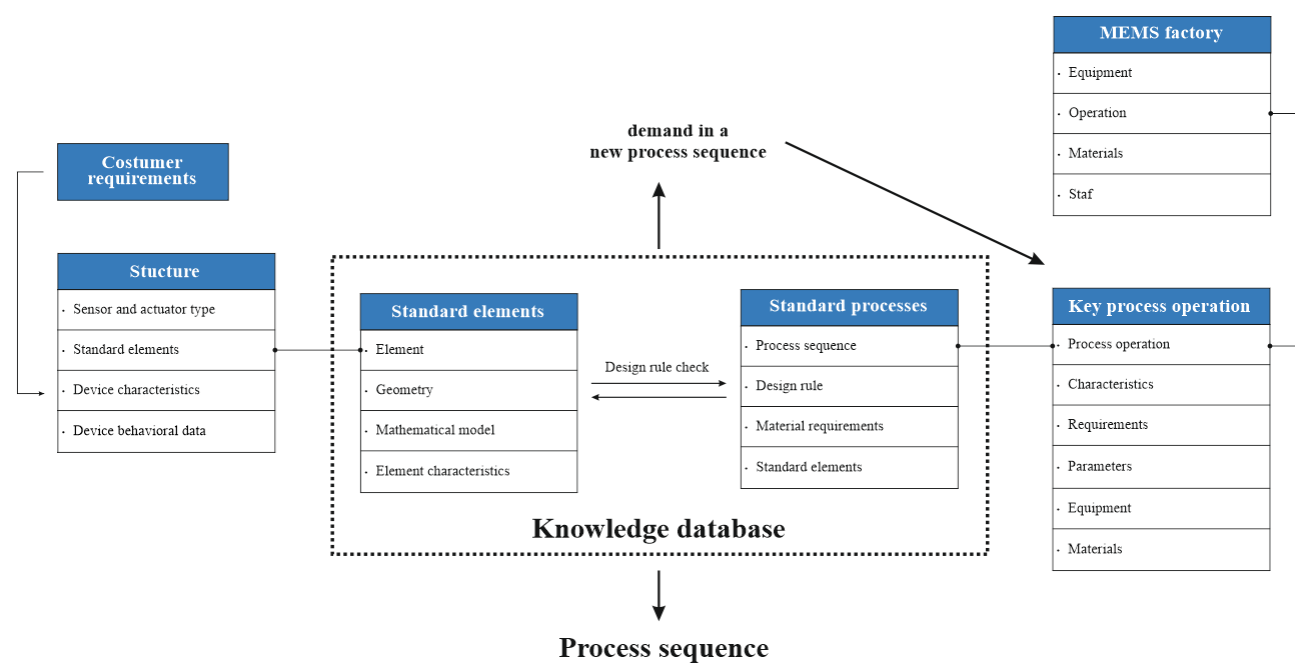

Fig. 13. Key elements of the MEMS digital modeling knowledge base.

The interaction between the technological and structural sections in the knowledge base allows us to judge the feasibility of implementing a compatible process sequence for a certain MEMS structure.

\section{Conclusion}

A comparative analysis of new product development principles in microelectronics, mechanical engineering, and in the production of MEMS is carried out. As a result, a MEMS integrated digital modeling approach is proposed based on the development of a knowledge base, including a description of the standard structural elements and the standard approved process sequences for their manufacture and the interactions between them.

\section{References}

1. i-SCOOP. https://www.i-scoop.eu/

2. i-Mcronews. https://www.i-micronews.com/

3. E. Mouner, How Megatrends will impact Mems\&Sensors, Technical report, Yole development (2018). 
4. C. Mead, L. Conway, Introduction to VLSI systems(Book), Addison-Wesley Publishing Co., 1980. 426 p. (1980).

5. J. Schlosser, Development and Verification of fast $\mathrm{C} / \mathrm{C}++$ Models for the Star12 Micro Controller (2001).

6. O.A. Al-Shebeeb, Analysis of the Integration of DFM Techniques and Effective Machining Parameter Selection in Metal Parts Manufacturing, West Virginia Univeristy (2019).

7. A.I. Borovkov, Yu.A. Ryabov, V.M. Maruseva, The new paradigm of digital design and modeling of globally competitive next-generation products, Digital production: methods, ecosystems, technologies, pp. 24-44 (2018).

8. J.R. Hauser et al, The House of Quality, Harvard Business Review. May-June. - 1997. pp. 63-73 (1988).

9. A.A. Samah, Integration of preference analysis methods into Quality Function Deployment, Cottbus: Springer (2011).

10. N.O. Erdil, O.M. Arani, Quality function deployment: more than a design tool, International Journal of Quality and Service Sciences (2019).

11. C.S. Smith, The manufacturing advisory service web based process and material selection, University of California, Berkeley (1999).

12. Y. Chen, S.K. Gupta, S. Feng, A Web-based process/material advisory system, Proceedings of IMECE00: ASME International Mechanical Engineering Congress and Exposition, Orlando, FL, pp. 5-10 (2000).

13. E.K. Antonsson, Structured design methods for MEMS final report, A Workshop sponsored by the National Science Foundation (1995).

14. R. Watty, Methodik zur Produktentwicklung in der Mikrosystemtechnik, Universität Stuttgart (2006).

15. K.L. Lamers, Components of an improved design process for micro-electromechanical systems, Stanford University (2009).

16. S.V. Uchaikin et al., Development of high-level design methods for multicomponent microsystems, Scientific and Technical Report, Application Code «2012-1.5-14-0002016-006», Tomsk (2013).

17. G.K. Fedder, Structured design methodology for MEMS, IEEE MEMS, vol. 99, pp. 1721, (1996).

18. R. Ghodssi, P. Lin, (Eds.), MEMS materials and processes handbook, vol. 1, Springer Science \& Business Media (2011).

19. S.E. Alper, MEMS gyroscopes for tactical-grade inertial measurement applications, The Graduate School of Natural and Applied Sciences of Middle East Technical University, Middle East Technical University,331 (2005). 